\section{International Scientific Journal Theoretical \& Applied Science}

p-ISSN: 2308-4944 (print)

e-ISSN: 2409-0085 (online)

Year: 2015 Issue: 02 Volume: 22

Published: 28.02.2015 http://www.T-Science.org
Georgy Vasil'evich Tokmazov

Associate professor,

Candidate of Pedagogical Science,

Professor Department of Mathematics, State Maritime University Admiral Ushakov,

Russia,

tokmazov@mail.ru

SECTION 21. Pedagogy. Psychology. Innovation in

Education.

\title{
DEVELOPMENT OF CRITERION RELIABILITY RESEARCH SKILLS IN THE PROCESS OF LEARNING MATHEMATICS
}

Abstract: Mathematical modeling of the dynamics of the criterion of reliability of research skills in the process of learning mathematics with accounting attempts to analyze research activities relative to the flow of the training error and the degree of compliance of the simulated entirety major research skills.

Key words: mathematical simulation, dynamics of development, research skills, the flow of the training error, the criterion of reliability.

Language: Russian

Citation: Tokmazov GV (2015) DEVELOPMENT OF CRITERION RELIABILITY RESEARCH SKILLS IN THE PROCESS OF LEARNING MATHEMATICS. ISJ Theoretical \& Applied Science 02 (22): 25-28. doi: http://dx.doi.org/10.15863/TAS.2015.02.22.6

\section{УДК 372.851 \\ РАЗВИТИЕ КРИТЕРИЯ НАДЕЖНОСТИ ИССЛЕДОВАТЕЛЬСКИХ УМЕНИЙ В ПРОЦЕССЕ ИЗУЧЕНИЯ МАТЕМАТИКИ}

Аннотация: Предложено математическое моделирование динамики развития критерия надёжности исследовательских умений в процессе изучения математики с учётом попытки анализа исследовательских действий относительно потока учебных ошибок и степени соответствия моделируемым ијелостным исследовательским умениям.

Ключевые слова: математическое моделирование, динамика развития, исследовательские умения, поток учебных ошибок, критерий надёжности

\begin{abstract}
Дальнейшее совершенствование процесса формирования исследовательских умений ориентируется на психологическую теорию деятельности, целостный анализ учебного знания, теорию формирования интеллекта. Одним из важных характеристик полученных результатов учебного процесса является степень соответствия моделируемым целостным исследовательским умениям. Это выражается в интегральном показателе учебного процесса - критерии надёжности полученного результата $[1 ; 2 ; 3]$.

При анализе критерия надёжности исследовательского умения в процессе изучения математике учитываются попытки исследовательских действий относительно потока учебных ошибок. Можно представить взаимосвязь между количеством учебных ошибок
\end{abstract}

и временем на их переформирование. То есть возникает параметр потока ошибок $\omega(t)$ и время на его переформирования $t_{c p}[4 ; 5]$.

Назовём параметром потока ошибок исследовательских умений величину равную отношению числа возникающих ошибок в единицу учебного времени к общему числу правильно выполненных исследовательских умений в единицу учебного времени:

$$
\omega(t)=\frac{n(\Delta t)}{(N \Delta t)},
$$

где $n(\Delta t)$ число исследовательских ошибок в интервале учебного времени от $t-\frac{\Delta t}{2}$ до $t+\frac{\Delta t}{2}$ 
; $N$ - общее число исследовательских умений; $\Delta t$ - интервал учебного времени. Параметр потока ошибок является статистической величиной $[6 ; 7 ; 8]$.

Параметр потока исследовательских ошибок и частота исследовательских ошибок для ординарных исследовательских потоков с ограниченным повторным действием можно представить интегральным уравнением Вольтера второго рода

$$
\omega(t)=a(t)+\int_{0}^{t} \omega(\tau) a(t-\tau) d \tau .
$$

По начальному значению потока исследовательских ошибок $a(t)$ можно определить количественные характеристики надёжности несформированных исследовательских умений. Поэтому (2) является основным уравнением, связывающим количественные характеристики надёжности несформированным и сформированных исследовательских умений при мгновенной исследовательской реакции $[9 ; 10 ; 11]$.

Данные закономерности можно представить в операторной форме

$$
\omega(s)=\frac{a(s)}{1-a(s)}, \quad a(s)=\frac{\omega(s)}{1+\omega(s)} .
$$

Данные соотношения могу определить взаимосвязь между выделенными характеристиками, если существуют преобразования Лапласа функций $a(s)$ и $\omega(s)$, а так же обратные преобразования данных выражений.

Параметр потока исследовательских ошибок $a(t)$ обладает следующими свойствами:

1) во всякий момент времени независимо от закона распределения времени безошибочной исследовательской деятельности параметр потока исследовательских ошибок больше, чем частота ошибок, то есть $\omega(t)>a(t)$;

2) независимо от вида функции потока исследовательских ошибок $a(t)$ параметр потока отказов $\omega(t)$ при $t \rightarrow \infty$ стремится к $\frac{1}{T_{c p}}$; это свойство параметра исследовательских ошибок показывает, что при длительной исследовательской учебной деятельности поток её ошибок независимо от закона распределения времени безошибочной работы становится стационарным, при этом интенсивность ошибок не является величиной постоянной;
3) если интенсивность исследовательских учебных ошибок $\lambda(t)$ - возрастающая функция времени, то $\lambda(t)>\omega(t)>\alpha(t), \quad$ если интенсивность исследовательских учебных ошибок $\lambda(t)$ - убывающая, функция времени то $\lambda(t)<\omega(t)<\alpha(t)$

4) при интенсивности исследовательских учебных ошибок $\lambda(t) \neq$ const параметр потока исследовательских учебных ошибок не равен сумме параметров потока отказов исследовательских элементов

$$
\omega_{c}(t) \neq \sum_{i=1}^{N} \omega_{i}(t) .
$$

Это свойство параметра потока исследовательских учебных ошибок устанавливает, что при вычислении количественных характеристик надёжности сложной исследовательской учебной деятельности, как системы, нельзя суммировать имеющиеся в настоящее время значения интенсивности ошибок исследовательских умений, полученные по статистическим данным об ошибках исследовательских учебных действий в условиях исследовательской учебной деятельности, так как указанные величины являются фактически параметрами потока ошибок;

5) при интенсивности исследовательских учебных ошибок $\lambda(t)=\lambda=$ const параметр потока исследовательских учебных ошибок равен интенсивности исследовательских учебных ошибок $\omega(t)=\lambda(t)=\lambda$.

Из анализа свойств интенсивности и параметра исследовательских учебных умений следует, что данные характеристики различны.

В настоящее время применяются статистические данные об исследовательских учебных ошибках, полученные в процессе учебной деятельности. При этом бывают случаи фиксации исследовательских учебных ошибок, что приводимые характеристики надёжности исследовательских учебных умений являются не интенсивностью ошибок, а параметрами потока ошибок $\omega(t)$. Это приводит к неверным результатам при расчёте надёжности исследовательских умений [12; 13; 14].

1) Для получения интенсивности ошибок исследовательских умений из статистических данных об ошибках исследовательской учебной деятельности в целом необходимо применять формулу (1), для чего важно знать процесс формирования и развития исследовательского умения. Это позволит адекватно, с высокой точностью совершенствовать методику отбора статистических данных о системе исследовательских учебных ошибках. Поэтому интенсивность исследовательских учебных 
ошибок $\lambda(t)$ целесообразно определять по параметру интенсивности исследовательских учебных ошибок $\omega(t)$. Методика расчёта определяется совокупностью вычислительных действий:

1) по статистическим данным об ошибках учебной исследовательской деятельности и по формуле (1) вычисляется поток ошибок и строится гистограмма $\omega_{i}(t)$;

2) гистограмма заменяется кривой, которая аппроксимируется уравнением;
3). Устанавливаются преобразования Лапласа $\omega_{i}(s)$ функции $\omega_{i}(t)$.

Это позволяет установить динамику развития критерия надёжности исследовательского умения в процессе изучения математики с учётом попытки анализа исследовательских действий относительно потока учебных ошибок $[15 ; 16]$.

\section{References:}

1. Mishchik SA (2014) Pedagogometrika and mathematical modeling educational activity. Materialy Mezhdunarodnoy nauchnoy konferenctsii "Modern mathematics in science" - 30.06.2014. ISJ Theoretical \&Applied Science 6(14): 54-56 Caracas, Venezuela. doi: http://dx.doi.org/10.15863/TAS.2014.06.14.10

2. Mishchik SA (2014) Simulation training activity methods of mathematical logic. Materialy Mezhdunarodnoy nauchnoy konferenctsii "Eurapean Science and Education" - 30.07.2014. ISJ Theoretical \&Applied Science 6(15): 72-74 Marseille, France. doi: http://dx.doi.org/10.15863/TAS.2014.07.15.13

3. Mishchik SA (2014) Mathematical modeling system integrity-cycle of life activity - first goal pedagogometriki. Materialy Mezhdunarodnoy nauchnoy konferenctsii "European Applied Sciences" - 30.08.2014. ISJ Theoretical \&Applied Science 7(16): 77-79 Aix-enProvence, France. doi: http://dx.doi.org/10.15863/TAS.2014.08.16.13

4. Mishchik SA (2014) Mathematical modeling system integrity-curricular activities - the second problem pedagogometriki. Materialy Mezhdunarodnoy nauchnoy konferenctsii "European Innovation" - 30.09.2014. ISJ Theoretical \&Applied Science 9(17): 126-128 Martigues, France. doi: http://dx.doi.org/10.15863/TAS.2014.09.17.21

5. Mishchik SA (2014) Mathematical modeling holistic-systemic communicative activity - the third task pedagogometriki. Materialy Mezhdunarodnoy nauchnoy konferenctsii "European Scientific Achievements" 30.10.2014. ISJ Theoretical \&Applied Science 10(18): 45-47 Brighton, UK. doi: http://dx.doi.org/10.15863/TAS.2014.10.18.11
6. Mishchik SA (2014) Mathematical modeling integrity - system performance subject - fourth task pedagogometriki. Materialy Mezhdunarodnoy nauchnoy konferenctsii "Eurapean Science and Technology" 30.11.2014. ISJ Theoretical \&Applied Science 11(19): 51-54 Southampton, United Kingdom. doi:

http://dx.doi.org/10.15863/TAS.2014.11.19.10

7. Mishchik SA (2014) Tselostno-sistemnyy tsikl uchebnoy zhiznedeyatel'nosti - model' professional'noy deyatel'nosti shirokoprofil'nogo spetsialista $\mathrm{V}$ sbornike: Deyatel'nostnaya teoriya ucheniya: sovremennoe sostoyanie i perspektivy. Materialy Mezhdunarodnoy nauchnoy konferentsii. Otvetstvennye redaktory: Yu.P. Zinchenko, O.A. Karabanova, A.I. Podol'skiy, G.A. Glotova. Moscow., 2014.- pp. 352-354

8. Tokmazov GV (2014) Matematicheskoe modelirovanie $\mathrm{v}$ uchebno-professional'noy deyatel'nosti. Materialy Mezhdunarodnoy nauchnoy konferenctsii "Modern mathematics in science" - 30.06.2014. ISJ Theoretical \&Applied Science 6(14): 44-46 Caracas, Venezuela. doi: http://dx.doi.org/10.15863/TAS.2014.06.14.8

9. Tokmazov GV (2014) Analysis says study skills in the study of mathematics. Materialy Mezhdunarodnoy nauchnoy konferenctsii "Eurapean Science and Education" 30.07.2014. ISJ Theoretical \&Applied Science 7(15): 72-74 Marseille, France. doi: http://dx.doi.org/10.15863/TAS.2014.07.15.14

10. Tokmazov GV (2014) Mathematical modeling research skills in educational activity methods of probability theory. Materialy Mezhdunarodnoy nauchnoy konferenctsii "Eurapean Science and Technology" - 
30.11.2014. ISJ Theoretical \&Applied Science 11(19): 66-69 Southampton, United Kingdom. doi:

http://dx.doi.org/10.15863/TAS.2014.11.19.13

11. Tokmazov GV (1994) Zadachi dinamicheskogo kharaktera. Matematika v shkole. - 1994. - № 5.- pp.9-12.

12. Tokmazov GV (1999) Ukrupnenie didakticheskikh edinits $\mathrm{v}$ zadachakh po teorii veroyatnostey. Matematika v shkole. - 1999. № 4.- pp.81-85.

13. Tokmazov GV (1999) Model' formirovaniya issledovatel'skikh umeniy na osnove trekhkomponentnoy strukture umstvennogo deystviya. Nauchnye trudy Moskovskogo pedagogicheskogo gosudarstvennogo universiteta. Seriya: Estestvennye nauki. Moscow: Prometey, 1999. - pp.68-88.

14. Tokmazov GV (2013) Sistematizatsiya i differentsirovannyy podkhod pri obuchenii resheniyu zadach nachal teorii veroyatnostey: monografiya. - 2-e izd., dop.i isprav. Novorossiysk: GMU imeni admirala F.F.Ushakova, 2013. - 176 p. - ISBN 978-589426-071-6

15. Tokmazov GV (2014) Opredelenie usloviy podgotovki uchashchikhsya $\mathrm{k}$ organizatsii issledovatel'skikh umeniy pri obuchenii matematike. Kazanskaya nauka. 2014. № 7. pp. 180-185.

16. Tokmazov GV (2014) MATHEMATICAL MODELING OF RESEARCH SKILLS METHODS OF PETRI NETS. Materialy Mezhdunarodnoy nauchnoy konferenctsii "Eurapean Science and Technology" 30.11.2014. ISJ Theoretical \&Applied Science 12 (20): 42-46 Birmingham, United Kingdom. doi:

http://dx.doi.org/10.15863/TAS.2014.12.20.10 